\title{
Effect of JAK2/STAT3 signaling pathway on liver injury associated with severe acute pancreatitis in rats
}

\author{
MINLI LI, XIAOHUA ZHANG, BIN WANG, XIAOBING XU, XIAOWEI WU, \\ MEIXIA GUO and FANGYU WANG
}

Department of Gastroenterology, Nanjing Medical University, Jinling Hospital, Nanjing, Jiangsu 210002, P.R. China

Received January 23, 2018; Accepted June 22, 2018

DOI: $10.3892 / \mathrm{etm} .2018 .6433$

\begin{abstract}
Janus kinase/signal transducers and activators of transcription (JAK/STAT) signaling constitutes one of the major pathways for cytokine signal transduction. However, the role of the JAK2/STAT3 pathway in liver injury during severe acute pancreatitis (SAP) remains unclear. The aim of this study was to investigate the role of the JAK2/STAT3 signaling pathway in liver injury after SAP. In the present study 64 male Sprague-Dawley rats were randomly divided into four groups: Control, AG490 (inhibition of JAK2), SAP and SAP with AG490. SAP was induced by retrograde infusion of $4 \%$ sodium taurocholate into the biliopancreatic duct. The activities of amylase (AMY) and liver enzymes were measured in serum. Livers and pancreas were isolated for measurements of histological damage. Blood and liver samples were taken for the measurement of TNF- $\alpha$, IL- 6 and IL-18 concentrations. The expression levels of JAK2 and STAT3 in liver tissue were detected by immunohistochemical staining and western blotting. The results demonstrated that amylase and liver enzymes were higher in the SAP groups compared with the control, AG490 and AG490-treated groups. The serum levels of TNF- $\alpha$, IL- 6 and IL-18 were effectively increased in the SAP groups, whereas they were reduced by AG490. Interestingly, JAK2 and STAT3 protein expression levels were significantly increased following induction of SAP and were significantly decreased in the AG490-pretreated groups. Administration of AG490 decreased the activity of pro-inflammatory cytokines and significantly attenuated SAP associated-liver injury in the rats. These results suggested that the mechanism may relate to the inhibition of TNF- $\alpha$, IL- 6 and IL-18, and inhibiting
\end{abstract}

Correspondence to: Professor Fangyu Wang, Department of Gastroenterology, Nanjing Medical University, Jinling Hospital, 305 Zhongshan Road, Nanjing, Jiangsu 210002, P.R. China

E-mail: wangfy65@nju.edu.cn

Key words: JAK/STAT pathway, AG490, servere acute pancreatitis, liver injury, inflammation excessive JAK2 and STAT3 activation, and may play a crucial role in the liver injury associated with SAP.

\section{Introduction}

Severe acute pancreatitis (SAP) is an exceptionally grave disorder of the viscera in the abdominal cavity, in which pancreatic elastases and a variety of other pro-inflammatory mediators are released into the portal and systemic circulation, leading to multiple organ failure and high rates of morbidity and mortality $(1,2)$. Although the precise mechanisms by which diverse etiological factors induce an attack remain unclear, once the disease process is initiated, common inflammatory and repair pathways are invoked (3). Significant attention has been dedicated to the investigation of acute lung injury in the setting of SAP, less attention has been devoted to pancreatitis-associated liver injury. The liver is also a major organ involved in the systemic inflammatory response and subsequent distant organ dysfunction observed during SAP. Previous studies have demonstrated the importance of the liver in the development of organ damage associated with SAP, specifically its role in extra pancreatic organ impairment following the release of macrophage-derived cytokines that play a critical role in the pathogenesis of pancreatitis and the subsequent inflammatory response (4-6). In this regard, the liver is a unique organ because Kupffer cells are the largest population of fixed-tissue macrophages and have been demonstrated to release pro-inflammatory cytokines (e.g., TNF- $\alpha$, IL-6 and IL-18) in response to local tissue and multiple organ damage (7). Thus, the pathogenesis of SAP-associated liver injury needs to be elucidated. Multiple studies have demonstrated that the Janus kinase/signal transducers and activators of transcription (JAK/STAT) signaling pathway is activated by pro-inflammatory cytokines, growth factors and hormones, and shown the beneficial and protective role of JAK2 and STAT3 in the inflammatory responses $(8,9)$. Previous studies have indicated that the soluble factors depend on hepatic STAT3 during systemic inflammation (10). However, the influence of the systemic inflammatory response on the JAK2/STAT3 signaling pathway in SAP is not well understood. In addition, the precise mechanisms of JAK2/STAT3 pathway inhibitors in $\mathrm{SAP}$ in relation to the degree of pancreatic diseases and liver 
injury remain largely unknown. The present study established an SAP rat model, examined the effects of the JAK2/STAT3 signaling pathway and investigated the possible protective effects of AG490 against SAP in rats.

\section{Materials and methods}

Animals. A total of 64 male Sprague-Dawley rats weighing between 200-250 g were used in the present study. The animals were purchased from Institutional Animal Care and Use Committee of Jinling Hospital (Nanjing, China). All operations were performed according to international guidelines concerning the care and treatment of experimental animals. Ethical approval for this study was obtained from the Ethics Committee for Animal Research at Jinling Hospital.

Animals were housed in standard conditions, in a temperature-controlled environment with a 12/12 light/dark cycle. Rats were randomly divided into four groups: Control $(\mathrm{n}=8)$, AG490 groups $(\mathrm{n}=8)$, SAP groups $(\mathrm{n}=24)$ and SAP-AG490 groups $(n=24)$. The SAP groups and SAP-AG490 groups were randomly divided into 6,12 and $18 \mathrm{~h}$ time-point groups, with 8 rats in each group. The controls received injections of $0.9 \%$ saline $(0.2 \mathrm{ml} / \mathrm{min})$ (11). AG490 groups received injections of AG490 dissolved in DMSO (30 nmol/ml; cat. no. sc-202046; Sigma-Aldrich; Merck KGaA, Darmstadt, Germany) via the vena caualis at a dose of $8 \mathrm{mg} / \mathrm{kg}$ with a single administration at $30 \mathrm{~min}$. SAP groups was induced by $4 \%$ sodium taurocholate in distilled water $(0.1 \mathrm{mg} / 100 \mathrm{~g}$ of body weight; Sigma-Aldrich; Merck KGaA) and retrograde infused at a rate of $20 \mu \mathrm{l} / \mathrm{min}$ using microinfusion pump continuously. SAP-AG490 groups were injected with AG490 before SAP model. The experiment was done half an hour with each rat after laparotomy. The rats were sequentially sacrificed 6,12 or $18 \mathrm{~h}$ after SAP induction with an intraperitoneal injection of pentobarbital $(200 \mathrm{mg} / \mathrm{kg}$; Shanghai Xinyu Biotech Co., Ltd., China); blood, liver and pancreatic tissues were collected for subsequent analysis.

Measurement of amylase (AMY) and liver enzymes activities. All blood samples were collected from the post cava of each animal. The serum AMY, alanine aminotransferase (ALT), and aspartate aminotransferase (AST) activities were determined in accordance with the manufacturer's protocols (Beijing Leadman Biochemistry Technology, Co., Ltd., China).

Analysis of TNF- $\alpha, I L-6$ and $I L-18$ in serum. Serum levels of TNF- $\alpha$ (cat. no. RTA00), IL-6 (cat. no. CATR6000B) and IL-18 (cat. no. DY992; all R\&D Systems Europe, Ltd., Abingdon, UK) concentrations were measured by ELISA kits in accordance with the manufacturer's protocols. All samples were determined in duplicate. The pro-inflammatory cytokines levels were expressed as $\mathrm{pg} / \mathrm{ml}$. Serum samples were collected and stored at $-80^{\circ} \mathrm{C}$ for subsequent biochemical and cytokine assays.

Reverse transcription-quantitative polymerase chain reaction (RT-qPCR) analysis of TNF- $\alpha, I L-6$ and $I L-18$ in liver tissue. Total RNA samples were isolated TRIzol reagent (cat. no. 15596-018; Invitrogen; Thermo Fisher Scientific, Inc., Waltham, MA, USA) from the rat liver tissues in accordance with the manufacturer's protocols. Complementary DNA
(cDNA) synthesis was performed using the cDNA reverse transcription kit (cat. no. AR0063; Wuhan Boster Biological Technology, Ltd., Wuhan, China). The primers (Sangon Biotech Co., Ltd., Shanghai, China) were synthesized as follows: TNF- $\alpha$ forward, 5'-TCAGTTCCATGGCCCAGA C-3' and reverse, 5'-GTTGTCTTTGAGATCCATGCC ATT-3'; IL-6 forward, 5'-AATCTGCTCTGGTCTTCTTGG AG-3' and reverse, 5'-GTTGGATGGTCTTGGTCCTTA G-3'; IL-18 forward, 5'-ACTGTACAACCGGAGTAATAC GG-3' and reverse, 5'-TCCATCTTGTTGTGTCCTGG-3', $\beta$-actin forward, 5'-CTGGCACCACACCTTCTACAATG-3' and reverse, 5'-AATGTCACGCACGATTTCCCGC-3'). The expression of target genes was normalized to that of $\beta$-actin. The samples were amplified in triplicate, and the results were calculated using the $2^{-\Delta \Delta C t}$ method.

Histopathological analysis. Pancreas and liver tissues were rinsed and weighed. The samples were collected from each rat and fixed in $4 \%$ neutral formalin immediately. The tissues were stained with hematoxylin-eosin (H\&E) by a trained pathologist. The morphologic evaluation of the tissues was performed by optical microscopy. The histological assessments of tissues were analyzed for SAP severity based on edema, inflammation and hemorrhage, and necrosis. The pathological scores of pancreas grading were performed as described by Schmidt et al (12). Liver pathological scores were evaluated according to the method reported by Camargo et al (13).

Immunohistochemical analysis. The method was used to detect expression of JAK2 and STAT3 proteins in liver tissue. The rats' livers were fixed with $4 \%$ paraformaldehyde for $24 \mathrm{~h}$ and then embedded in paraffin. After being deparaffinized and pretreated in citrate buffer, sections were incubated with rabbit anti-rat JAK2 (1:200; cat. no. 3230) and STAT (1:200; cat. no. 330835; both Cell Signaling Technology, Inc., Danvers, MA, USA) primary antibodies with PBS at $4^{\circ} \mathrm{C}$ overnight. After three washes with PBS, the liver sections were incubated with isothiocyanate-conjugated goat anti-rabbit antibody (1:200; cat. no. BA1010; Wuhan Boster Biological Technology, Ltd.) for $15 \mathrm{~min}$ at room temperature and then rinsed in PBS. The negative control for the immunostaining were incubated with rabbit $\operatorname{IgG}$ without the specific primary antibody. To measure JAK2 and STAT3 proteins, 5 fields were randomly selected and analyzed using Image-Pro Plus software version 6.0 (Media Cybernetics, Inc., Rockville, MD, USA) at a magnification, $\mathrm{x} 200$.

Western blot analysis of JAK2, p-JAK2, STAT3 and p-STAT3 in liver tissues. Western blot analysis was performed to measure the JAK2, phosphorylated-JAK2 (p-JAK2; cat. no. 3776), STAT3 and phosphorylated-STAT3 (p-STAT; cat. no. 39145S; both Cell Signaling Technology, Inc.) protein expression levels in all the groups. Total proteins were extracted from the frozen liver samples using ice-cold RIPA buffer containing protease inhibitors. Protein concentration was quantified by BCA Protein Assay kit (Beyotime Institute of Biotechnology, Haimen, China). Then the protein samples were separated by SDS-PAGE and transferred onto PVDF membranes. The samples were incubated in 5\% nonfat milk in TBS with $0.1 \%$ Tween for $1 \mathrm{~h}$, and with primary antibodies 
Table I. Serum levels of AMY, ALT and AST in the different groups (U/L, n=8, $\bar{x} \pm s)$.

\begin{tabular}{lccc}
\hline Group & AMY & ALT & AST \\
\hline Control & $545.11 \pm 23.52$ & $24.29 \pm 3.17$ & $19.23 \pm 2.39$ \\
AG490 & $558.22 \pm 31.76$ & $28.36 \pm 6.25$ & $21.28 \pm 3.74$ \\
SAP-6 h & $1,247.45 \pm 156.37^{\mathrm{a}}$ & $156.64 \pm 17.48^{\mathrm{a}}$ & $276.39 \pm 27.44^{\mathrm{a}}$ \\
SAP-12 h & $3,478.26 \pm 212.58^{\mathrm{a}}$ & $198.49 \pm 21.36^{\mathrm{a}}$ & $396.45 \pm 30.27^{\mathrm{a}}$ \\
SAP-18 h & $4,461.64 \pm 346.17^{\mathrm{a}}$ & $352.23 \pm 29.18^{\mathrm{a}}$ & $478.46 \pm 31.57^{\mathrm{a}}$ \\
SAP-AG490-6 h & $1,582.16 \pm 125.34^{\mathrm{a}}$ & $117.56 \pm 16.37^{\mathrm{a}, \mathrm{b}}$ & $324.36 \pm 23.78^{\mathrm{a}, \mathrm{b}}$ \\
SAP-AG490-12 h & $3,201.56 \pm 199.29^{\mathrm{a}}$ & $155.82 \pm 24.56^{\mathrm{a}, \mathrm{b}}$ & $320.69 \pm 29.74^{\mathrm{a}, \mathrm{b}}$ \\
SAP-AG490-18 h & $3,719.74 \pm 287.63^{\mathrm{a}, \mathrm{b}}$ & $287.33 \pm 19.63^{\mathrm{a}, \mathrm{b}}$ & $386.48 \pm 28.83^{\mathrm{a}, \mathrm{b}}$ \\
\hline
\end{tabular}

No changes of AMY, ALT and AST were observed in the control and AG490 groups. Levels of AMY, ALT and AST increased significantly under SAP conditions. AG490 decreased the effects in SAP-AG490 groups. P $>0.05$ control vs. AG490 groups. ${ }^{a}$ P $<0.05$ vs. control groups. ${ }^{\mathrm{b}} \mathrm{P}<0.05$ for multiple comparisons of SAP and treatment groups at the same time point. AMY, amylase; ALT, alanine aminotransferase; AST, aspartate aminotransferase; SAP, severe acute pancreatitis.

(rabbit anti-rat JAK2, p-Jak2, STAT3 and p-STAT3; 1:1,000) overnight at $4^{\circ} \mathrm{C}$. The blots were washed and incubated with appropriate goat anti-rabbit antibodies and an enhanced chemiluminescence detection assay used according to the manufacturer's instructions. $\beta$-actin was used as a loading control. All assays were independently repeated three times to assure reproducibility of results. The positive target bands were quantified by Quantity One software (Bio-Rad Laboratories, Inc., Hercules, CA, USA).

Statistical analysis. The statistical analyses were performed using the Statistical Package for the Social Sciences (SPSS) software version 17.0 (SPSS, Inc., Chicago, IL, USA). Data are expressed as means \pm SD. Differences between groups were compared using one-way analysis of variance (ANOVA) and followed by Bonferroni's multiple comparison test. $\mathrm{P}<0.05$ was considered to indicate a statistically significant difference. All possible comparisons were made between different groups.

\section{Results}

The serum levels of AMY, ALT and AST. No changes in AMY, ALT or AST were observed in the control and AG490 groups. The activity levels of AMY, ALT and AST in the SAP groups increased significantly at 6,12 and $18 \mathrm{~h}$ time points compared with the control and AG490 groups $(\mathrm{P}<0.05)$. Compared with SAP groups, the activity levels of AMY, ALT and AST were suppressed in the SAP-AG490 groups at different time points $(\mathrm{P}<0.05$; Table I).

Expression of TNF- $\alpha, I L-6$ and IL-18 levels in the serum and liver tissues. It is well known that pro-inflammatory cytokines are important signaling molecules in the development of SAP. To evaluate the effects of pro-inflammatory cytokine production and to further determine the mechanisms that underlie reduced injury following AG490 pretreatment, the expression of TNF- $\alpha$, IL- 6 and IL-18 in serum and liver tissues were identified by ELISA and RT-qPCR. The levels of TNF- $\alpha$, IL-6 and IL-18 were similar in the control and AG490 groups. As indicated by ELISA and RT-qPCR, these cytokines increased markedly in the SAP groups compared with the control and AG490 groups at each time point $(\mathrm{P}<0.05)$. It was then determined whether inhibition of the pro-inflammatory response by AG490 was mediated through the JAK2/STAT3 signaling pathway. Compared with SAP groups, these cytokines were suppressed by AG490 pretreatment in the SAP-AG490 groups, but AG490 alone did not have a significant effect on the cytokine levels in AG490 groups ( $\mathrm{P}<0.05$; Table II, Fig. 1).

The histopathological alterations of the pancreas. To determine the changes in the experimental SAP model, the pathological examination results of the pancreas of rats at different experimental groups were observed using an optical microscope. The results indicated that the pancreatic tissue were normal in the control and AG490 groups $(\mathrm{P}<0.05)$. While in the SAP groups, a large number of inflammatory cells infiltrated the pancreas. The histopathological changes, pancreatic edema and substantial necrosis, appeared more pronounced and worsened significantly with time. There were also differing degrees of damage in the SAP-AG490 groups, but less severe damage in the SAP groups $(\mathrm{P}<0.05$; Fig. 2).

Histopathological alterations of the liver. The scoring was used to determine the degree of liver injury. Livers of the control and AG490 groups showed normal structure. Compared with the control and AG490 groups, liver cell edema, inflammatory cell infiltration, hepatic spotty necrosis and coagulation necrosis were detected in the SAP groups. However, the SAP-AG490 groups exhibited significant attenuation of these changes, and the edema, necrosis and inflammatory reaction were significantly reversed compared with the SAP groups $(\mathrm{P}<0.05$; Fig. 3$)$.

Immunohistochemistry analysis of JAK2 and STAT3. To detect the degree of inflammation in liver tissues, immunostaining of JAK2 (Fig. 4) and STAT3 (Fig. 5) were performed in the liver samples at the time points for each group. In the control and AG490 groups, minimal staining of JAK2 and STAT3 was observed in the liver lining. However, expression of JAK2 and STAT3 were increased, with JAK2 protein appearing mainly 
Table II. Serum levels of TNF- $\alpha$, IL-6, IL-18 in the different groups (pg/ml, n=8, $\bar{x} \pm s)$.

\begin{tabular}{lccc}
\hline Group & TNF- $\alpha$ & IL-6 & IL-18 \\
\hline Control & $60.78 \pm 3.81$ & $44.23 \pm 4.73$ & $23.50 \pm 2.17$ \\
AG490 & $54.12 \pm 8.43$ & $42.37 \pm 5.96$ & $24.61 \pm 2.99$ \\
SAP-6 h & $161.25 \pm 10.02^{\mathrm{a}}$ & $337.45 \pm 39.64^{\mathrm{a}}$ & $145.05 \pm 31.40^{\mathrm{a}}$ \\
SAP-12 h & $253.05 \pm 13.24^{\mathrm{a}}$ & $487.47 \pm 38.41^{\mathrm{a}}$ & $352.23 \pm 20.50^{\mathrm{a}}$ \\
SAP-18 h & $346.78 \pm 35.18^{\mathrm{a}}$ & $599.36 \pm 51.29^{\mathrm{a}}$ & $468.86 \pm 21.32^{\mathrm{a}}$ \\
SAP-AG490-6 h & $150.01 \pm 22.25^{\mathrm{a}, \mathrm{b}}$ & $310.27 \pm 27.68^{\mathrm{a}, \mathrm{b}}$ & $122.57 \pm 11.63^{\mathrm{a}, \mathrm{b}}$ \\
SAP-AG490-12 h & $228.37 \pm 16.57^{\mathrm{a}, \mathrm{b}}$ & $367.56 \pm 38.12^{\mathrm{a}, \mathrm{b}}$ & $305.45 \pm 27.95^{\mathrm{a}, \mathrm{b}}$ \\
SAP-AG490-18 h & $299.13 \pm 21.33^{\mathrm{a}, \mathrm{b}}$ & $401.92 \pm 40.73^{\mathrm{a}, \mathrm{b}}$ & $402.37 \pm 26.58^{\mathrm{a}, \mathrm{b}}$ \\
\hline
\end{tabular}

Serum TNF- $\alpha$, IL- 6 and IL-18 levels were determined using ELISA. The serum obtained following the induction of pancreatitis showed increased amounts of pro-inflammatory cytokines and this production was blocked by AG490. P $>0.05$ control vs. AG490 groups. ${ }^{\mathrm{a} P}<0.05$ vs. control groups. ${ }^{b} \mathrm{P}<0.05$ for multiple comparisons of $\mathrm{SAP}$ and treatment groups at the same time point. SAP, severe acute pancreatitis.

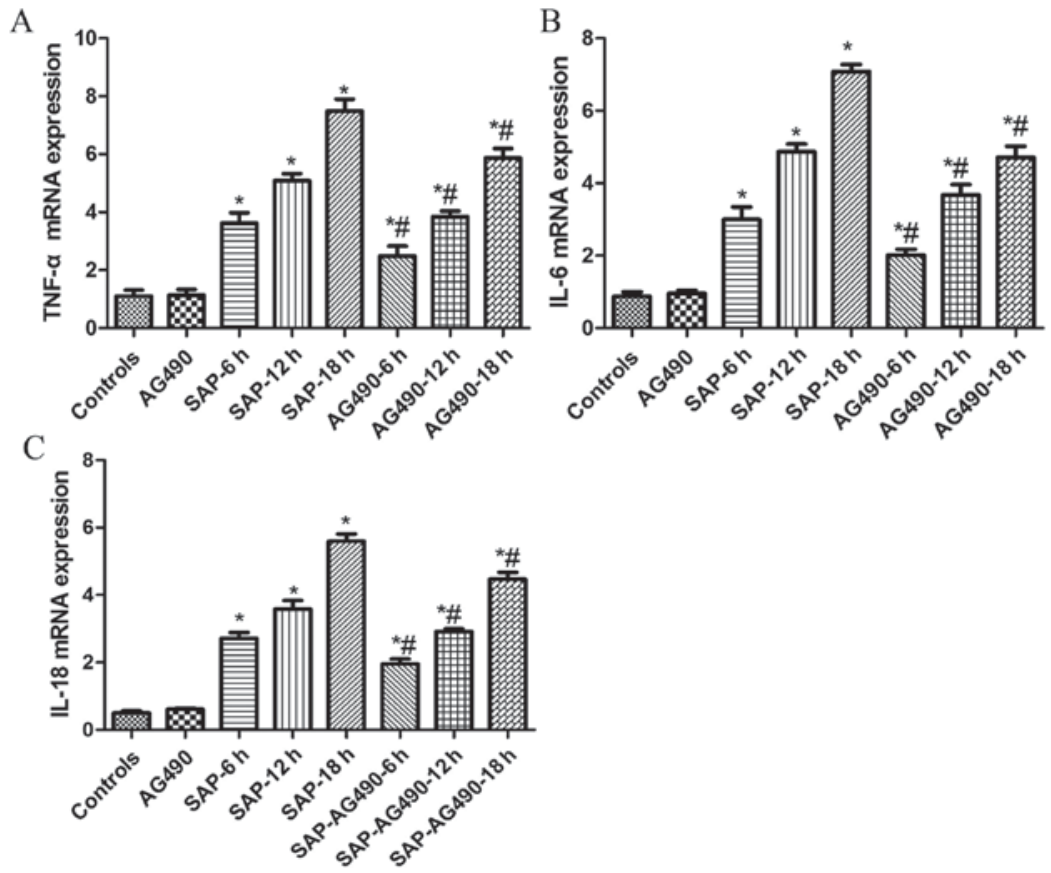

Figure 1. Expression of TNF- $\alpha$, IL-6 and IL-18 in liver tissues. (A) TNF- $\alpha$; (B) IL-6; and (C) IL-18. No difference was observed between the control and AG490 groups $(\mathrm{P}>0.05)$. As demonstrated in SAP groups at each time point, the levels of TNF- $\alpha$, IL- 6 and IL-18 were significantly increased compared with the control and AG490 groups, respectively, and were significantly down regulated by AG490 ( $\mathrm{P}<0.05)$. $\mathrm{P}>0.05$ control vs. AG490 groups; " $\mathrm{P}<0.05$ vs. control groups; ${ }^{\# P}<0.05$ vs. SAP groups at each time point. SAP, severe acute pancreatitis.

in the cytoplasm and STAT3 protein appearing mainly in the nucleus. The proportions of cytoplasmic JAK2 and nuclear STAT3 in SAP groups were significantly increased compared with the control and AG490 groups. By contrast, the expression of these proteins in SAP-AG490 groups was significantly lower compared with SAP groups at each time point $(\mathrm{P}<0.05)$.

Expression of JAK2, p-JAK2, STAT3 and p-STAT3 in rat liver tissues determined by western blot analysis. As shown in Fig. 5, the activation of JAK2 and STAT3 was examined in liver tissues and the changes in the p-JAK2 and p-STAT3 levels in these tissues were measured. Western blot analysis showed that no change was found in AG490 groups compared with control $(\mathrm{P}>0.05)$. The expressions of JAK2, p-JAK2,
STAT3 and p-STAT3 proteins in SAP groups and SAP-AG490 groups at $6,12,18 \mathrm{~h}$ were markedly higher compared with control and AG490 groups $(\mathrm{P}<0.05)$. As further confirmation of the role of the JAK2/STAT3 pathway in the liver tissues, the SAP-AG490-pretreated groups showed a reduction in the JAK2, p-JAK2, STAT3 and p-STAT3 levels compared with SAP groups at the same time point $(\mathrm{P}<0.05$; Fig. 6$)$.

\section{Discussion}

SAP is characterized by its acute onset and rapid progression, leading to clinical multiple organ dysfunction syndromes with systemic inflammation and high mortality. A high range of pancreatic injury is found in the early stages of liver failure. 
A

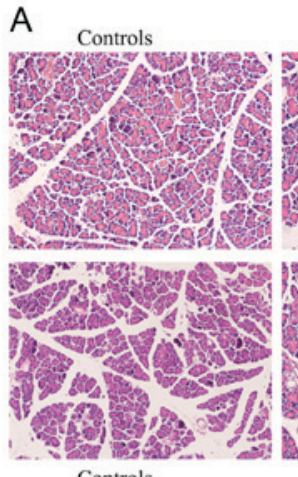

SAP-6 $\mathrm{h}$

SAP- $12 \mathrm{~h}$

SAP- $18 \mathrm{~h}$
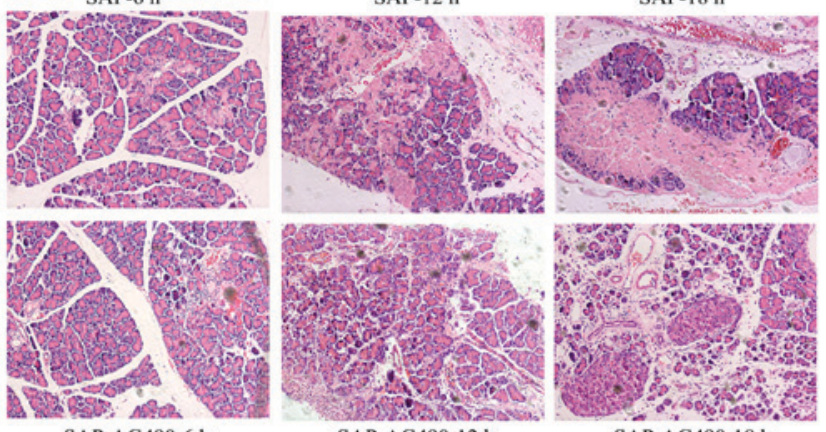

SAP-AG490-6 h
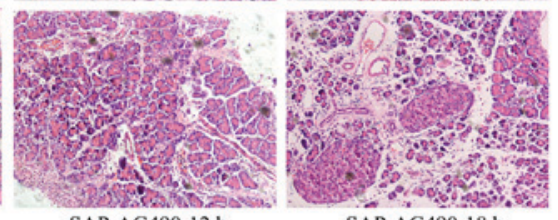

B

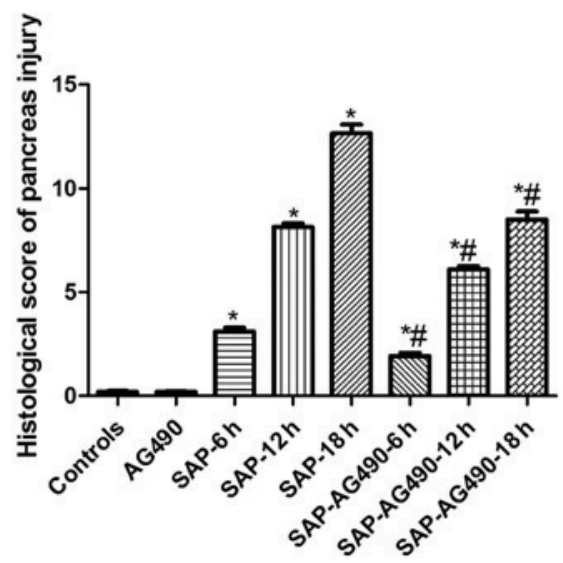

SAP-AG490-18 h

Figure 2. Histopathological changes of the pancreas in rats from each group (magnification, x200). (A) Representative H\&E staining images of pancreatic tissues from each group of rats. (B) Histological score of pancreas injury. Pancreas of Control and AG490 groups showed normal structure. All SAP groups had pancreatic edema, inflammatory cell infiltration, fat necrosis and extensive hemorrhaging which increased gradually and became more serious at the 12 and $18 \mathrm{~h}$ time points. Both SAP-AG490 treatment rat edema and necrosis was significantly reduced compared with the SAP groups at each time point. P $>0.05$ control vs. AG490 groups; ${ }^{\mathrm{P}}<0.05$ vs. control groups; ${ }^{\prime} \mathrm{P}<0.05$ vs. SAP groups at each time point. SAP, severe acute pancreatitis; H\&E, hematoxylin-eosin.

A

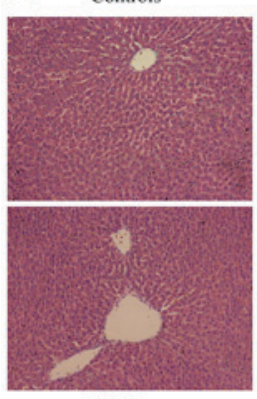

AG490
SAP-6 h
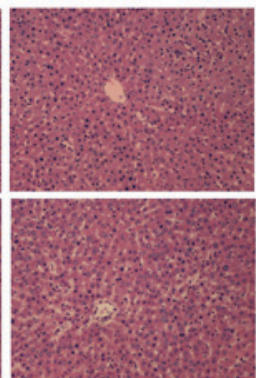

SAP-AG490 $-6 \mathrm{~h}$

B
SAP- 12 h
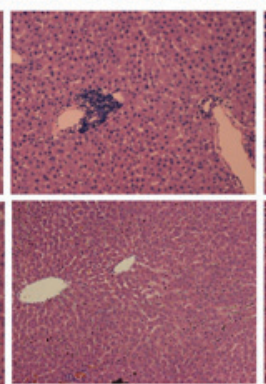

SAP-AG490 - $12 \mathrm{~h}$

SAP-18 h

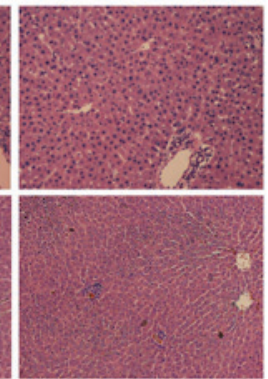

SAP-AG490-18 h

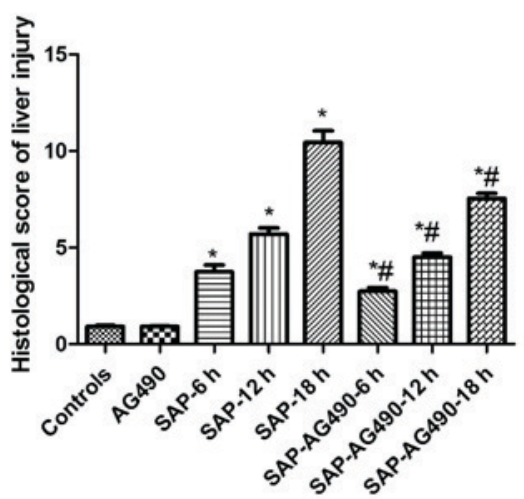

Figure 3. Histopathological changes in the livers of rats from each group (magnification, x200). (A) Representative H\&E staining images of liver tissues from each group of rats. (B) Histological score of liver injury. The structure of liver tissue was clear and showed normal architecture in the control and AG490 groups, while the bridging necrosis and loosened cytoplasm showed in all SAP groups. SAP-AG490 treatment groups showed significantly reduced periportal and lobular inflammatory infiltration. $\mathrm{P}>0.05$ control vs. AG490 groups; " $\mathrm{P}<0.05$ vs. control groups; $\mathrm{P}<0.05$ for multiple comparisons of SAP and treatment groups at each time point. SAP, severe acute pancreatitis; $\mathrm{H} \& \mathrm{E}$, hematoxylin-eosin. 

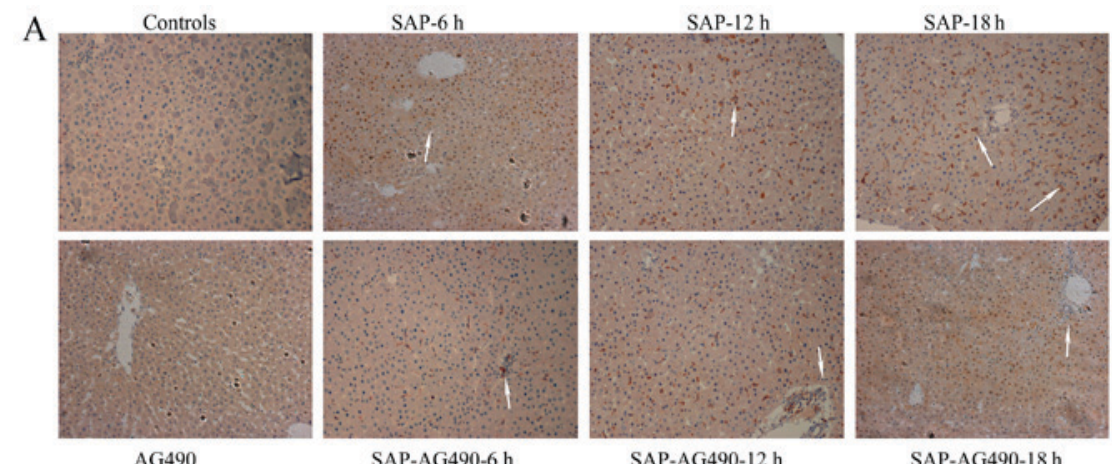

SAP-AG490-12 h
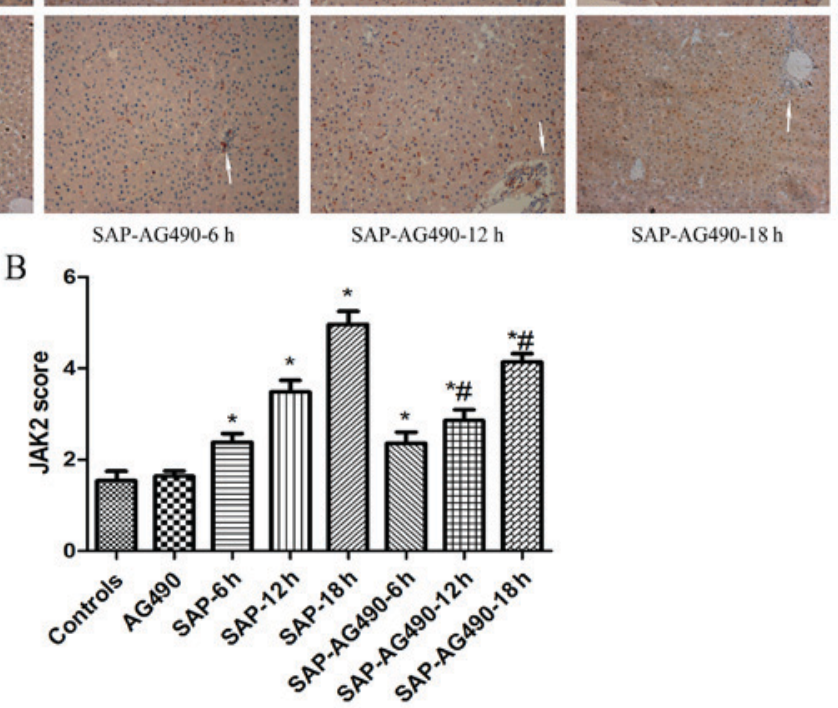

SAP-AG490-18 h

Figure 4. Immunohistochemical localization of JAK2 in the liver tissues (magnification, x200). (A) Immunohistochemical analysis of JAK2 expression in liver tissues from each group. (B) Graphical analysis of JAK2 expression in each group. The arrows indicated JAK2 expressed in the cytoplasm. The expression of JAK2 was weakly detected in the cytoplasm. In SAP groups, JAK2 was markedly over expressed in the cytoplasm at 6, 12 and $18 \mathrm{~h}$ time points. With AG490 treatment, the expression of JAK2 was markedly decreased in liver tissues. $\mathrm{P}>0.05$ control vs. AG490 groups; ${ }^{*} \mathrm{P}<0.05$ vs. control groups; ${ }^{*} \mathrm{P}<0.05$ for multiple comparisons of SAP and treatment groups at each time point. SAP, severe acute pancreatitis; JAK, Janus kinase.

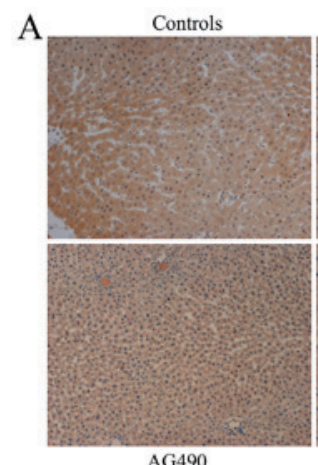

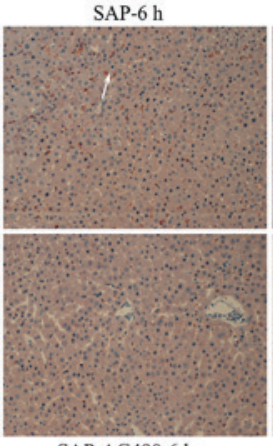

SAP-AG490-6 h

$\mathrm{B}$

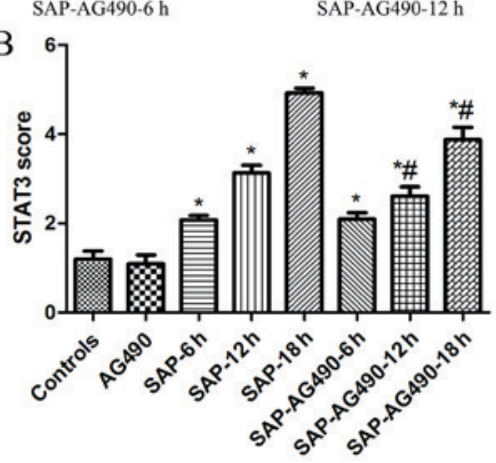

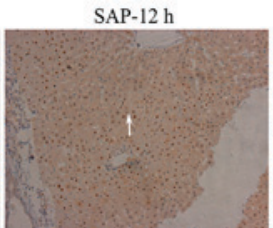
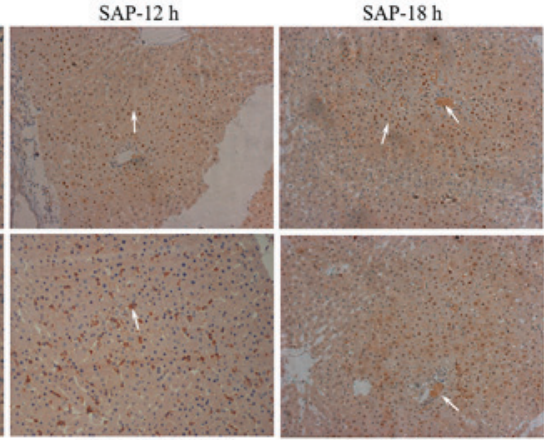

SAP-AG490-18 h

Figure 5. Immunohistochemical localization of STAT3 in the liver tissues (magnification, x200). (A) Immunohistochemical analysis of STAT3 expression in liver tissues from each group. (B) Graphical analysis of STAT3 expression in each group. The arrows indicated STAT3 expressed in the nucleus. In the control groups, the expression of STAT3 was weakly observed in the nucleus. In SAP groups, intense positive staining of STAT3 increased markedly at 6, 12 and $18 \mathrm{~h}$ time points. AG490-treated liver tissues showed a reduction of STAT3 in the nucleus. P $>0.05$ control vs. AG490 groups; ${ }^{*} \mathrm{P}<0.05$ vs. control groups; ${ }^{\#} \mathrm{P}<0.05$ vs. SAP groups at each time point. SAP, severe acute pancreatitis; STAT, signal transducers and activators of transcription.

It is associated with the release of digestive enzymes into the pancreatic interstitium, as well as inflammatory responses, leading to pancreatitis and subsequent distant organ dysfunction due to the excessive production and systemic spreading 
A
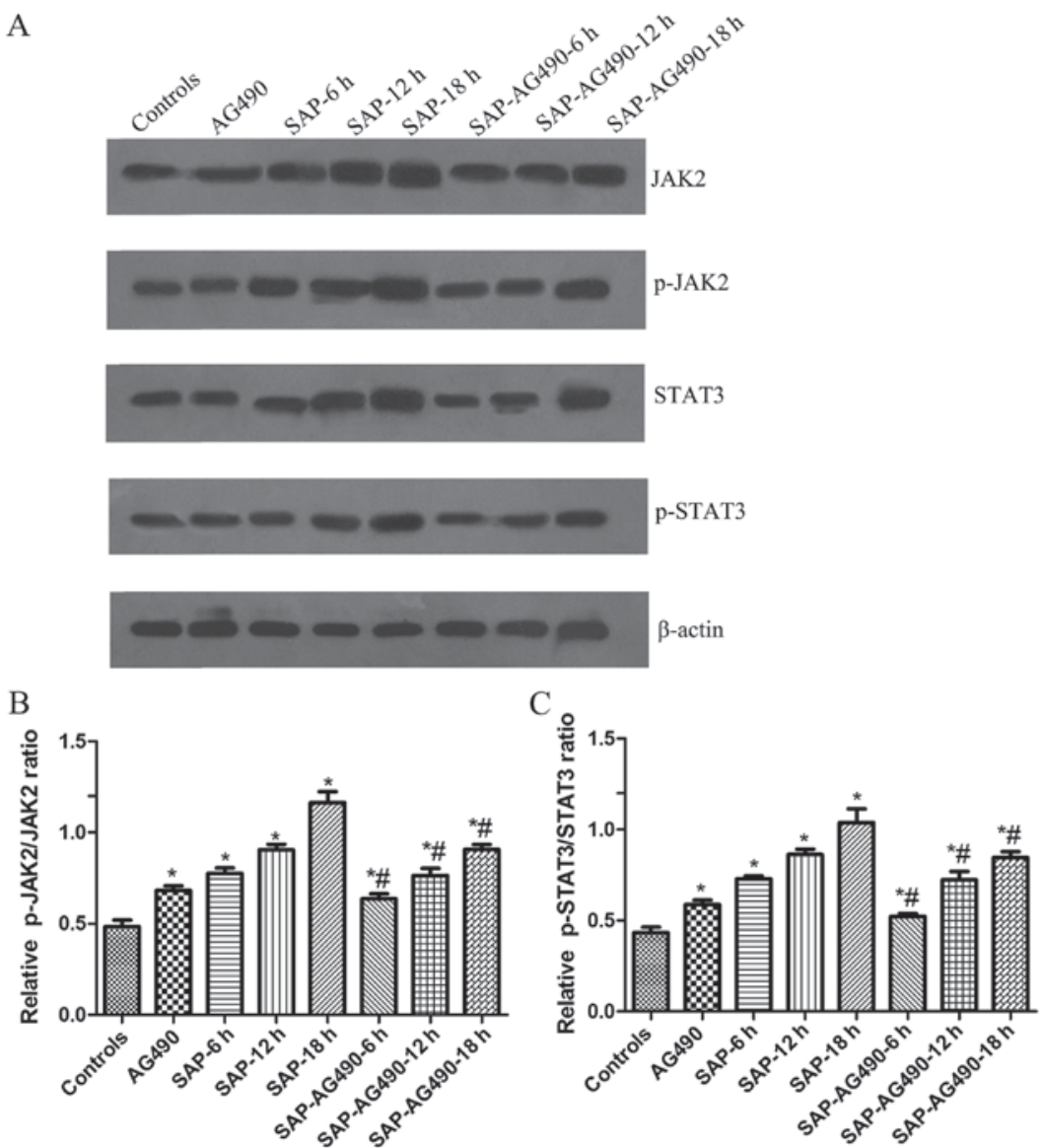

Figure 6. Representative western blot analysis of JAK2, p-JAK2, STAT3 and p-STAT3 proteins at different time points in the livers. The graphs show densitometric analysis of the western blots. $\beta$-actin was used as the internal reference. (A) Quantitative analysis of the protein of JAK2, p-JAK2, STAT3 and p-STAT3 in liver tissues; (B) Statistical analysis of p-JAK2/JAK2 ratio and (C) p-STAT3/STAT3 ratio. P $>0.05$ control vs. AG490 groups. "P<0.05 vs. control groups. ${ }^{\#} \mathrm{P}<0.05$ vs. SAP groups at each time point. SAP, severe acute pancreatitis; JAK, Janus kinase; STAT, signal transducers and activators of transcription.

of inflammatory mediators $(4,14,15)$. TNF- $\alpha$, IL-6 and IL-18 have been shown to play important roles in mediating SAP and liver injury (16-18). In particular, liver injury is a severe complication of SAP, resulting in systemic inflammation and an increased risk of mortality (19). Although previous studies have suggested that activation of the JAK/STAT pathway up-regulated the expression of pro-inflammatory cytokines in the progression of SAP (20-22), the molecular mechanisms underlying their roles in mediating acute liver injury during the course of SAP remain poorly understood.

The JAK/STAT signaling pathway is widely involved in inflammation (23), and numerous studies have shown that the JAK and STAT family of kinases activate the cytokine signaling cascade. Among the most important protein tyrosine kinase families, JAK2 and STAT3 were identified from studies on the downstream events of cytokine receptor binding, including the transcriptional activation of genes involved in inflammatory responses $(24,25)$. Studies have shown that JAK and STAT activation occurs widely throughout the pancreas and liver in inflammatory cells (26-29). Specifically, cytokines trigger the activation of STAT3 mediated by JAK2; additionally, JAK2 plays an important role in the activation of STAT3, as observed in the liver. Another study has indicated that TNF- $\alpha$ activated JAK2 and STAT1 or STAT3 on the development of pancreatic injury in vitro (30). Blocking the JAK1/STAT1 signaling pathway prevents the lethal effects of excessive systemic inflammatory response during SAP (31). However, the influence of the systemic inflammatory response on the JAK2/STAT3 signaling pathway in SAP and its relation to the degree of pancreatic diseases is not well understood. Therefore, we explored these possible modulatory effects on the pro-inflammatory cytokines (TNF- $\alpha$, IL- 6 and IL-18)/JAK2 and STAT3 signaling pathway in liver tissues of SAP rats. The present study found that the expression of TNF- $\alpha$, IL- 6 and IL-18, and the severity of inflammatory response in SAP models significantly increased compared with the controls. In addition, the systemic manifestations of SAP were not only caused by local inflammatory processes but also by the excessive production and systemic spreading of inflammatory mediators. It was found that the transcription factors JAK2 and STAT3 were rapidly activated in the liver following sodium taurocholate injection, with the protein expressions of JAK2, p-JAK2, STAT3, and p-STAT3 in liver tissues increased significantly in SAP groups, especially at the $18 \mathrm{~h}$ time point group. This finding possibly indicates that pro-inflammatory cytokines can regulate the JAK2/STAT3 pathway.

AG490, a member of the tyrphostin family of protein kinase inhibitors, selectively inhibits the phosphorylation of JAK2 and STAT3. To eliminate these conflicting factors, we investigated the relationship between the production of pro-inflammatory 
cytokines and the activation of JAK2/STAT3 in pancreatic inflammation in vivo. Previous studies have demonstrated that TNF- $\alpha$ and IL- 6 are primarily produced by macrophages and play a major role in pancreatitis, and the reduction of these cytokines in patients may be useful in the treatment of AP-related diseases (32). In the pancreas, IL-18 is a pleiotropic cytokine that is secreted primarily by activated macrophages and Kupffer cells, and which stimulates the release of other pro-inflammatory cytokines (33-35). It has been reported that a high level of IL-18 is associated with the early phase of AP in in vivo models (36). The present study induced AP and administered pre-treatment with AG490 in different rat models. In the SAP groups at different time points, the rats exhibited a marked increase in AMY, ALT and AST concentrations. Moreover, it was found that histopathological changes in the pancreas and liver were amplified significantly with the administration of sodium taurocholate, as indicated by parenchyma necrosis, inflammatory infiltration and bleeding, suggesting that serious pancreas and liver injury had occurred, which is consistent with previous studies $(37,38)$. The present study revealed that pancreatic and liver injury became dramatically more serious with time, compared with the pathological changes in SAP rats to varying degrees. Similarly, the JAK2 inhibitor AG490 effectively inhibited the activation of JAK2 and STAT3 phosphorylation. Interestingly, JAK2 and STAT3 levels were affected by the overexpression of TNF- $\alpha$, IL- 6 and IL-18, as indicated by the high levels of these proteins in the liver tissues. The decrease in the TNF- $\alpha$, IL- 6 and IL-18 protein expression levels suggested that the anti-inflammatory effects of AG490 might be mediated through the JAK2/STAT3 signaling pathway. That IL-6 triggered JAK2 phosphorylation indicated a tightly restricted signaling pattern for JAK2. Moreover, pretreatment with AG490 produced prompt and marked decreases in JAK2/STAT3, which suggested an upstream requirement for JAK2 in this response, and inhibited JAK2 production by down-regulating pro-inflammatory cytokines and attenuating the activation of STAT3, decreasing the severity of pancreas and liver injury, and the inflammatory response. Thus, there is a strong association between JAK2/STAT3 activities and the invasive ability of rats, which suggests that inhibition of this pathway may lead to the identification of a valid target for the treatment of liver injury in SAP.

The present study demonstrated that activation of JAK2/STAT3 gene expression and production of inflammatory cytokines, such as TNF- $\alpha$, IL- 6 and IL-18, led to pancreatitis-induced liver injury. Therefore, these results indicated that strategies to protect liver functions at an early time point may help prevent multiple system organ failure and could also aid in determining the pathogenesis and appropriate treatment options for SAP. Further investigations could facilitate the early recognition of the development of systemic complications by clinicians and improve the management of SAP.

\section{Acknowledgements}

The present authors would like to thank the Anesthesiology Department of the Jinling Hospital for their generosity in supplying the JAK2 antibody.

\section{Funding}

The present study was supported by the Army Health Project (grant no. 14BJZ28).

\section{Availability of data and materials}

The datasets used and/or analyzed during the current study are available from the corresponding author on reasonable request.

\section{Authors' contributions}

ML, XZ and FW designed the research; ML, XX, XW, BW and $M G$ performed the research and obtained the data; $X X$ and MG provided the kits. ML wrote the paper; XZ and FW finalized and revised the manuscript. All authors have read and approved the final manuscript.

\section{Ethics approval and consent to participate}

The present study was approved by the Institutional Animal Care and Use Committee of Jinling Hospital. All operations were performed according to international guidelines concerning the care and treatment of experimental animals. Ethical approval for this study was obtained from the Ethics Committee for Animal Research at Jinling Hospital.

\section{Patient consent for publication}

Not applicable.

\section{Competing interests}

The authors declare that there is no conflict of interest related to the present study.

\section{References}

1. Shen HN, Lu CL and Li CY: Effect of diabetes on severity and hospital mortality in patients with acute pancreatitis: A national population-based study. Diabetes Care 35: 1061-1066, 2012.

2. Banks PA, Bollen TL, Dervenis C, Gooszen HG, Johnson CD, Sarr MG, Tsiotos GG and Vege SS; Acute Pancreatitis Classification Working Group: Classification of acute pancreatitis 2012: Revision of the atlanta classification and definitions by international consensus. Gut 62: 102-111, 2013.

3. Liu Y, Liao R, Qiang Z and Zhang C: Pro-inflammatory cytokine-driven PI3K/Akt/Sp1 signalling and $\mathrm{H}_{2} \mathrm{~S}$ production facilitates the pathogenesis of severe acute pancreatitis. Biosci Rep 37: BSR20160483, 2017.

4. Yang J, Gallagher SF, Haines K, Epling-Burnette PK, Bai F, Gower WR Jr, Mastorides S, Norman JG and Murr MM: Kupffer cell-derived Fas ligand plays a role in liver injury and hepatocyte death. J Gastrointest Surg 8: 166-174, 2004.

5. Ou ZB, Miao CM, Ye MX, Xing DP, He K, Li PZ, Zhu RT and Gong JP: Investigation for role of tissue factor and blood coagulation system in severe acute pancreatitis and associated liver injury. Biomed Pharmacother 85: 380-388, 2017.

6. Yang J, Fier A, Carter Y, Liu G, Epling-Burnette PK, Bai F, Loughran TP Jr, Mastorides S, Norman JG and Murr MM: Liver injury during acute pancreatitis: Therole of pancreatitis-associated ascitic fluid (PAAF), p38-MAPK, and caspase-3 in inducing hepatocyte apoptosis. J Gastrointest Surg 7: 200-208, 2003.

7. Folch-Puy E: Importance of the liver in systemic complications associated with acute pancreatitis: The role of Kupffer cells. J Pathol 211: 383-388, 2007. 
8. O'Shea JJ, Schwartz DM, Villarino AV, Gadina M, McInnes IB and Laurence A: The JAK-STAT pathway: Impact on human disease and therapeutic intervention. Annu Rev Med 66: 311-328, 2015.

9. Roskoski R Jr: Janus kinase (JAK) inhibitors in the treatment of inflammatory and neoplastic diseases. Pharmacol Res 111: 784-803, 2016

10. Sakamori R, Takehara T, Ohnishi C, Tatsumi T, Ohkawa K, Takeda K, Akira S and Hayashi N: Signal transducer and activator of transcription 3 signaling within hepatocytes attenuates systemic inflammatory response and lethality in septic mice. Hepatology 46: 1564-1573, 2007.

11. Aho HJ, Koskensalo SM and Nevalainen TJ: Experimental pancreatitis in the rat. Sodium taurocholate-induced acute haemorrhagic pancreatitis. Scand J Gastroenterol 15: 411-416, 1980.

12. Schmidt J, Rattner DW, Lewandrowski K, Compton CC, Mandavilli U, Knoefel WT and Warshaw AL: A better model of acute pancreatitis for evaluating therapy. Ann Surg 215: 44-56, 1992.

13. Camargo CA Jr, Madden JF, Gao W, Selvan RS and Clavien A Interleukin-6 protects liver against warm ischemia/reperfusion injury and promotes hepatocyte proliferation in the rodent. Hepatology 26: 1513-1520, 1997.

14. Kempuraj D, Twait EC, Williard DE, Yuan Z, Meyerholz DK and Samuel I: The novel cytokine interleukin-33 activates acinar cell proinflammatory pathways and induces acute pancreatic inflammation in mice. PLoS One 8: e56866, 2013.

15. Geisler F, Algül H, Riemann M and Schmid RM: Questioning current concepts in acute pancreatitis: Endotoxin contamination of porcine pancreatic elastase is responsible for experimental pancreatitis-associated distant organ failure. J Immunol 174 6431-6439, 2005

16. Jiang Y, An Y, Jiang D, Wu B, Yang Y and Sun D: TNF- $\alpha$ regulating interleukin-33 induces acute pancreatic inflammation in rats. Ann Clin Lab Sci 46: 54-59, 2016.

17. Martin MA, Saracibar E, Santamaria A, Arranz E, Garrote JA, Almaraz A, del Olmo ML, García-Pajares F, Fernández-Orcajo P, Velicia R, et al: Interleukin 18 (IL-18) and other immunological parameters as markers of severity in acute pancreatitis. Rev Esp Enferm Dig 100: 768-773, 2008 (In Spanish).

18. Zhang XH, Li ML, Wang B, Guo MX and Zhu RM: Caspase-1 inhibition alleviates acute renal injury in rats with severe acute pancreatitis. World J Gastroenterol 20: 10457-10463, 2014.

19. Wenhong D, Jia Y, Weixing W, Xiaoyan C, Chen C, Sheng X and Hao J: Zerumbone attenuates the severity of acute necrotizing pancreatitis and pancreatitis-induced hepatic injury. Mediators Inflamm 2012: 156507, 2012

20. Yang J, Gallagher SF, Haines K, Epling-Burnette PK, Bai F, Gower WR Jr, Mastorides S, Norman JG and Murr MM: Kupffer cell-derived Fas ligand plays a role in liver injury and hepatocyte death. J Gastrointest Surg 8: 166-174, 2004.

21. Chen P, Huang L, Zhang Y, Qiao M, Yao W and Yuan Y: The antagonist of the JAK-1/STAT-1 signaling pathway improves the severity of cerulein-stimulated pancreatic injury via inhibition of NF- $\kappa \mathrm{B}$ activity. Int J Mol Med 27: 731-738, 2011.

22. Damm J, Harden L, Gerstberger R, Roth J and Rummel C: The putative JAK-STAT inhibitor AG490 exacerbates LPS-fever, reduces sickness behavior, and alters the expression of pro- and anti-inflammatory genes in the rat brain. Neuropharmacology 71 98-111, 2013

23. Yu JH and Kim H: Role of janus kinase/signal transducers and activators of transcription in the pathogenesis of pancreatitis and pancreatic cancer. Gut Liver 6: 417-422, 2012.
24. Huang C, Ma R, Sun S, Wei G, Fang Y, Liu R and Li G: JAK2-STAT3 signaling pathway mediates thrombin-induced proinflammatory actions of microglia in vitro. J Neuroimmunol 204: 118-125, 2008.

25. Agrawal S, Gollapudi S, Su H and Gupta S: Leptin activates human B cells to secrete TNF- $\alpha$, IL-6, and IL-10 via JAK2/STAT3 and p38MAPK/ERK1/2 signaling pathway. J Clin Immunol 31: 472-478, 2011.

26. Huang LY, Chen P, Xu LX, Zhou YF, Zhang YP and Yuan YZ: Fractalkine upregulates inflammation through CX3CR1 and the Jak-Stat pathway in severe acute pancreatitis rat model. Inflammation 35: 1023-1030, 2012.

27. Gallmeier E, Schäfer C, Moubarak P, Tietz A, Plössl I, Huss R, Göke B and Wagner AC: JAK and STAT proteins are expressed and activated by IFN-gamma in rat pancreatic acinar cells. J Cell Physiol 203: 209-216, 2005.

28. Wu L, Li H, Zheng SZ, Liu X, Cai H and Cai BC: Da-Huang-Fu-Zi-Tang attenuates liver injury in rats with severe acute pancreatitis. J Ethnopharmacol 150: 960-966, 2013.

29. Yu JH, Kim KH and Kim H: SOCS 3 and PPAR-gamma ligands inhibit the expression of IL- 6 and TGF-betal by regulating JAK2/STAT3 signaling in pancreas. Int J Biochem Cell Biol 40: 677-688, 2008

30. Robinson K, Vona-Davis L, Riggs D, Jackson B and McFadden D: Peptide YY attenuates STAT1 and STAT3 activation induced by TNF-alpha in acinar cell line AR42J. J Am Coll Surg 202: 788-796, 2006

31. Yu JH, Kim KH and Kim H: Suppression of IL-1beta expression by the Jak 2 inhibitor AG490 in cerulein-stimulated pancreatic acinar cells. Biochem Pharmacol 72: 1555-1562, 2006

32. Surbatovic M and Radakovic S: Tumor necrosis factor- $\alpha$ levels early in severe acute pancreatitis: Is there predictive value regarding severity and outcome? J Clin Gastroenterol 47: 637-643, 2013

33. Gallagher SF, Peng Y, Haines K, Baksh K, Epling-Burnette PK, Yang J and Murr MM: Fas/FasL play a central role in pancreatitis-induced hepatocyte apoptotis. J Gastrointest Surg 9: 467-475, 2005

34. McCormack D, McDonald D and McFadden D: Pterostilbene ameliorates tumor necrosis factor alpha-induced pancreatitis in vitro. J Surg Res 178: 28-32, 2012.

35. Ueda T, Takeyama Y, Yasuda T, Matsumura N, Sawa H, Nakajima T, Ajiki T, Fujino Y, Suzuki Y and Kuroda Y: Significant elevation of serum interleukin-18 levels in patients with acute pancreatitis. J Gastroenterol 41: 158-165, 2006.

36. Ning JW, Zhang Y, Yu MS, Gu ML, Xu J, Usman A and Ji F: Emodin alleviates intestinal mucosal injury in rats with severe acute pancreatitis via the caspase-1 inhibition. Hepatobiliary Pancreat Dis Int 16: 431-436, 2017.

37. Segersvärd R, Tsai JA, Herrington MK and Wang F: Obesity alters cytokine gene expression and promotes liver injury in rats with acute pancreatitis. Obesity (Silver Spring) 16: 23-28, 2008.

38. Lv P, Fan LJ, Li HY, Meng QS and Liu J: Protective effect of thalidomide on liver injury in rats with acute pancreatitis via inhibition of oxidative stress. Ann Clin Lab Sci 45: 508-514, 2015.

This work is licensed under a Creative Commons Attribution-NonCommercial-NoDerivatives 4.0 International (CC BY-NC-ND 4.0) License. 